\title{
Fasudil Increases the Establishment of Somatic Cell Nuclear Transfer Embryonic Stem Cells in Mouse
}

\author{
Seongjun So, Mustafa Zafer Karagozlu, Yeonmi Lee and Eunju Kang* \\ Department of Convergence Medicine \& Stem Cell Center, Asan Institute for Life Sciences, Asan Medical Center, \\ University of Ulsan College of Medicine, Seoul 05505, Korea
}

Received December 5, 2019

Revised December 26, 2019

Accepted February 6, 2020

*Correspondence

Eunju Kang

E-mail: kangeun@amc.seoul.kr

ORCID

https://orcid.org/0000-0002-2830-6779
ABSTRACT Somatic cell nuclear transfer derived embryonic stem cells (NT-ESCs) have significant advantages in various fields such as genetics, embryology, stem cell science, and regenerative medicine. However, the poor establishment of NT-ESCs hinders various research. Here, we applied fasudil, a Rho-associated kinase (ROCK) inhibitor, to develop somatic cell nuclear transfer (SCNT) embryos and establish NTESCs. In the study, MII oocytes were isolated from female B6D2F1 mice and performed SCNT with mouse embryonic fibroblasts (MEFs). The reconstructed NT-oocytes were activated artificially, and cultured to blastocysts in KSOM supplemented with $10 \mu \mathrm{M}$ fasudil. Further, the blastocysts were seeded on inactivated MEFs in embryonic stem cell medium supplemented with $10 \mu \mathrm{M}$ fasudil. A total of $26 \%$ of embryos formed into blastocysts in the fasudil treated group, while this ratio was $44 \%$ in the fasudil free control group. On the other hand, 30\% of blastocysts were established NT-ESCs after exposure of fasudil, which was significantly higher than the control group (10\%). The results suggest that fasudil reduced blastocyst development after SCNT due to inhibition of 2 cell cleavage while improved the establishment of NT-ESCs through the anti-apoptotic pathway.

Keywords: embryo development, embryonic stem cells, fasudil, somatic cells nuclear transfer

\section{INTRODUCTION}

Embryonic stem cells (ESCs) can proliferate infinitely and able to differentiate into all cell types (Ying et al., 2008). Among them, pluripotent stem cells (PSCs) derived from somatic cells via nuclear transfer (NT-ESCs) provide a generation of autologous stem cells. The first success on NT-ESCs was reported in a bovine model and subsequently in mouse models (Kawase et al., 2000). Finally, human NT-ESCs were successfully developed (Tachibana et al., 2013). Today, human NT-ESCs are vital resources for patient-specific regenerative medicine to treat degenerative disorders such as diabetes, osteoarthritis, and parkinson's disease, without causing immune rejection because they have the same genetic information with the donor (Polejaeva and Campbell, 2000). Since NT-ESCs appear to have similar pluripotent characteristics such as transcription, genetics, epigenetics, differentiation, and self-renewal ability as those of conventional ESC lines, which were derived from embryos produced by fertilization (Roh, 2012; Ma et al., 2014), they are used effectively in broad fields such as genetics and embryology. However, until now, the rates for the development of NT-blastocysts from reconstructed embryos and the establishment of NT-ESCs are 
relatively low (Zhao et al., 2007)

The rho-associated kinase (ROCK) activates phosphomyosin light chain (pMLC), which contracts intracellular actomyosin and is known to induce apoptosis in various cells (Amano et al., 2010). Hence, the ROCK inhibitors, especially Y-27632, is widely used in the field of PSCs, including ESCs, NT-ESCs, and induced pluripotent stem cells (iPSCs) for increasing survival and proliferation (Watanabe et al., 2007). Fasudil is another ROCK inhibitor, which has been approved for clinical use and already used in Japan for the prevention of cerebral vasospasm after subarachnoid hemorrhage (Liu Guang Jian et al., 2012). It also suppresses the ROCK signal and increases the survival of many types of cells (Cavallini et al., 2003; Chen Meihui et al., 2013), and has been using in various cellular research such as culture of neural stem cells, differentiation of bone marrow mesenchymal stem cells into neuron-like cells, and cardiac differentiation of human pluripotent stem cells (Chen Shu et al., 2015; Cheng et al., 2015; Hu et al., 2019). However, fasudil had not been used in embryo research. In the present study, we investigated the effectiveness of fasudil in the development of the embryo into blastocysts and the establishment of NTmESCs.

\section{MATERIALS AND METHODS}

\section{Ethics and governance}

Experiments on live vertebrates were performed in compliance with institutional regulations of the Asan Institute for Life Sciences and were approved Asan Institutional Animal Care and Use Committee (IACUC: 2018-12-269). All experiments were performed in accordance with relevant guidelines and regulations.

\section{Treatment of fasudil}

The developmental phases divided into two groups, phase 1: from NT-oocyte to blastocyst stage and phase 2: from blastocyst to NT-ESC stage. For the investigation of effectiveness, fasudil was added to the culture medium at a concentration of $10 \mathrm{uM}$ during all developmental phases from NT to mESC based on the previous reports (Watanabe et al., 2007; So et al., 2019).

\section{Preparation of nuclear donor cells}

Isolation of mouse embryonic fibroblasts (MEFs) was performed as previously described (Kang et al., 2014). MEFs were cultured with DMEM/F12 (Life Technologies) supplemented with $10 \%$ fetal bovine serum (FBS, Gibco), $2 \mathrm{mM}$ glutamine (Life Technologies), $0.1 \mathrm{mM}$ nonessential amino acids (Life Technologies), $0.1 \mathrm{mM}$ 2-mercaptoethanol (2-ME, Sigma-Aldrich), $100 \mathrm{U} / \mathrm{mL}$ penicillin, 100 $\mathrm{mg} / \mathrm{mL}$ streptomycin (GE Life Science) and $10 \mu \mathrm{M}$ fasudil (Adooq) on gelatin-coated culture plates. The medium was changed once every three days.

mESCs were cultured using mES medium; KODMEM supplemented with 20\% Knockout Serum Replacement (KSR; Life Technologies), $1000 \mathrm{U} / \mathrm{mL}$ leukemia inhibitory factor (LIF; Millipore), $3 \mu \mathrm{M}$ CHIR99021 (Peprotech), $0.5 \mu \mathrm{M}$ PD0325901 (Peprotech), $2 \mathrm{mM}$ glutamine, $0.1 \mathrm{mM}$ nonessential amino acids, $0.1 \mathrm{mM} 2-\mathrm{ME}, 100 \mathrm{U} / \mathrm{mL}$ penicillin, and $100 \mathrm{mg} / \mathrm{mL}$ streptomycin on MEF feeder cells coated plates.

\section{Collection of oocytes}

Oocytes were collected from 6- to 8-week-old female B6D2F1 mice. For ovulation induction, $5 \mathrm{IU} / \mathrm{mL}$ pregnant mare serum gonadotropin (PMSG, Daesung Microbiological Labs. Co.) was injected into the abdominal cavity of female B6D2F1 mice (Leyva et al., 1998). After injection of PMSG (50 hrs), we injected $5 \mathrm{IU} / \mathrm{mL}$ human chorionic gonadotropin (HCG, Daesung Microbiological Labs. Co.) to the mice (Kang et al., 2014). Then, the mice sacrificed, and oviducts were isolated $13 \mathrm{hrs}$ after HCG injection. Cumulus-oocyte complexes were collected from oviduct using 26 gauge syringe (Korea Vaccine Co.) and transferred to a medium containing $0.1 \%$ hyaluronidase for dissociation of cumulus cells. Oocytes that were detached from cumulus cells were rinsed and incubated in KSOM (Millipore) at $37^{\circ} \mathrm{C}$ in $5 \% \mathrm{CO}_{2}$ before use.

\section{Somatic cell nuclear transfer (SCNT)}

MII oocytes from female B6D2F1 mice were placed into separate $10 \mu \mathrm{L}$ manipulation droplets of FHM medium containing $5 \mu \mathrm{g} / \mathrm{mL}$ cytochalasin B (CCB; Sigma-Aldrich) and $1.25 \mathrm{mM}$ caffeine (Sigma-Aldrich), in a glass-bottom dish. Donor cells were detached using $0.25 \%$ trypsin/ EDTA (Gibco) and resuspension in phosphate-buffered saline (PBS; Hyclone). Drops were covered with tissue culture oil and placed on the stage of the microscope (Nikon) with micromanipulators (Narishige), and a laser objective (Hamilton Thorne). The oocyte was fixed using 
a holding pipette, placing the spindle close to 5 o' clock position. The zona pellucida near the spindle was drilled with a laser, and an enucleation pipette was inserted to remove the spindle. After removing the spindle, the dissociated donor cell was mixed with HVJ-E Sendai virus $\left(G e n o m O N E^{\mathrm{TM}}\right.$ ) and injected to enucleated oocyte using the enucleation pipette (Kang et al., 2014).

\section{Artificial activation of oocytes and embryo culture}

NT-oocytes were transfer droplets of activation medium supplemented with $10 \mathrm{mM} \mathrm{SrCl}_{2}, 500 \mathrm{nM}$ scriptaid, and $5 \mu \mathrm{g} / \mathrm{mL} \mathrm{CCB}$, in the presence or absence of $10 \mu \mathrm{M} \mathrm{fa-}$ sudil diluted in dimethyl sulfoxide (DMSO; Sigma-Aldrich) (Moon et al., 2017). After cultured for 5 hrs at $37^{\circ} \mathrm{C}$ in $5 \%$ $\mathrm{CO}_{2}$, pseudo pronuclei ( $\mathrm{PN}$ ) formed oocytes were transferred to KSOM supplemented with or without $10 \mu \mathrm{M}$ fasudil, and incubated at $37^{\circ} \mathrm{C}$ in $5 \% \mathrm{CO}_{2}$.

\section{Establishment of NT-mESC lines}

Acid Tyrode's (AT) solution was used for the removal of zona pellucida as the assistance of blastocyst hatching on the assigned embryos (Neev et al., 1993). Blastocysts were transferred to AT solution drop on a petri dish. After zona pellucida disappeared completely, blastocysts were transferred to the mES medium drop for washing. Then, blastocysts were seeded on MEF coated 4-well plates filled with $\mathrm{mES}$ medium treated with or without fasudil (Tsung and Mummery, 1990). At this time, blastocysts divided into two groups according to inner cell mass quality and trophectoderm quality following criteria of the Gardner blastocyst grading system (Gardner et al., 2000). Also, the grouped cells divided into subgroups as, Inner cell mass quality; A: many cells, tightly packed, B: several cells, loosely grouped, C: very few cells, and Trophectoderm quality; A: many cells, forming a cohesive layer, B: few cells, forming a loose epithelium, C: very few large cells.

\section{Statistical analysis}

The $p$-value for statistical comparisons between the groups was calculated using Fisher's extract test. Prism 5 software (GraphPad) was used for the calculation of significance.

\section{RESULTS}

\section{Effects of fasudil on NT-embryo development}

To determine the effectiveness of fasudil in the development of NT-embryo, we treated $10 \mu \mathrm{M}$ fasudil to embryo culture medium after SCNT (Fig. 1). SCNT was performed

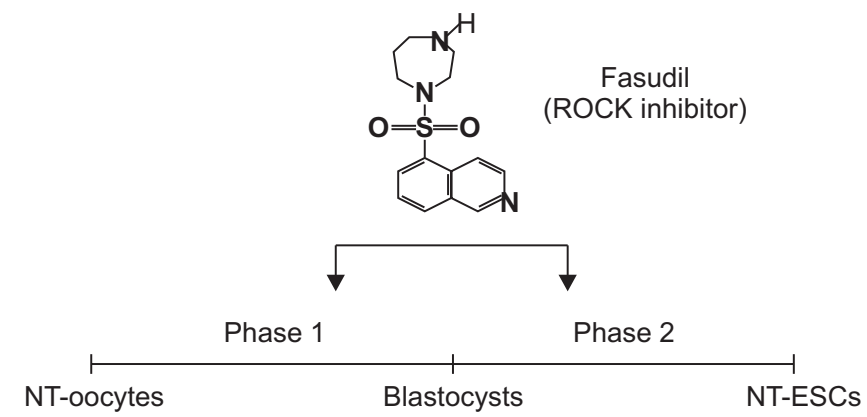

Fig. 1. Experimental design. Fasudil has treated two developmental stages. Phase 1: from reconsented NT-oocyte to blastocysts; Phase 2: seeded blastocysts on the MEFs. NT; nuclear transfer, MEF; mouse fetal fibroblast.
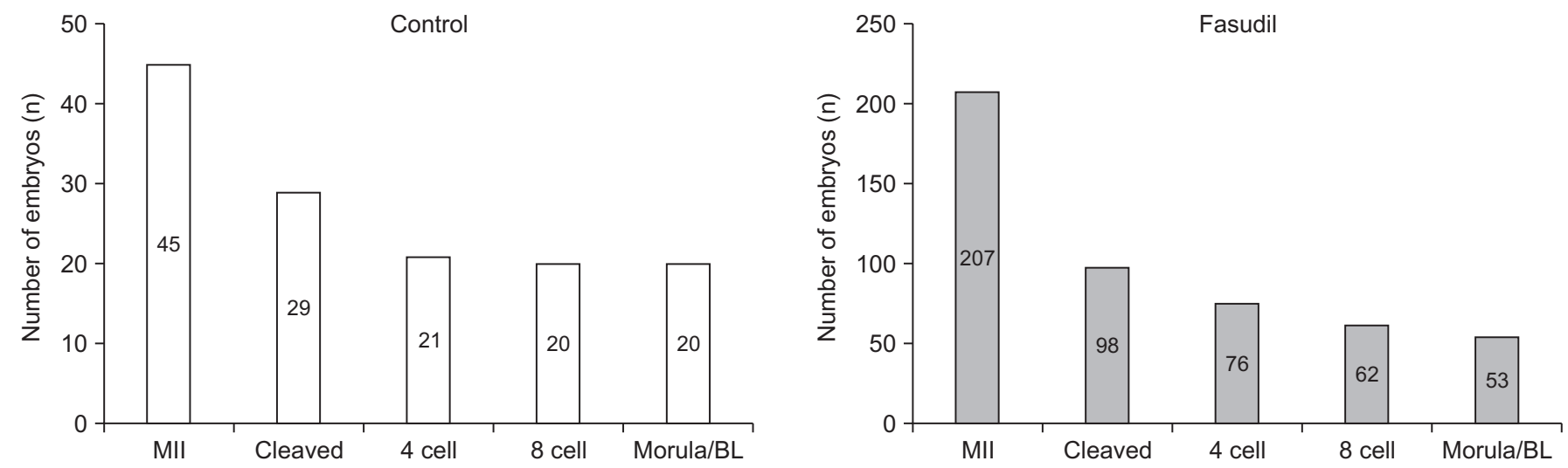

Fig. 2. The number of embryo development after SCNT. In the control group (without fasudil), 45 NT oocytes were used, and 20 blastocysts were formed (44\%), while 53 blastocysts were developed from 207 NT embryos with fasudil supplementation (26\%). Fasudil rather reduced blastocyst development. SCNT; somatic cell nuclear transfer, NT; nuclear transfer, PN; pseudo pronucleus, mESCs; mouse embryonic stem cells. 


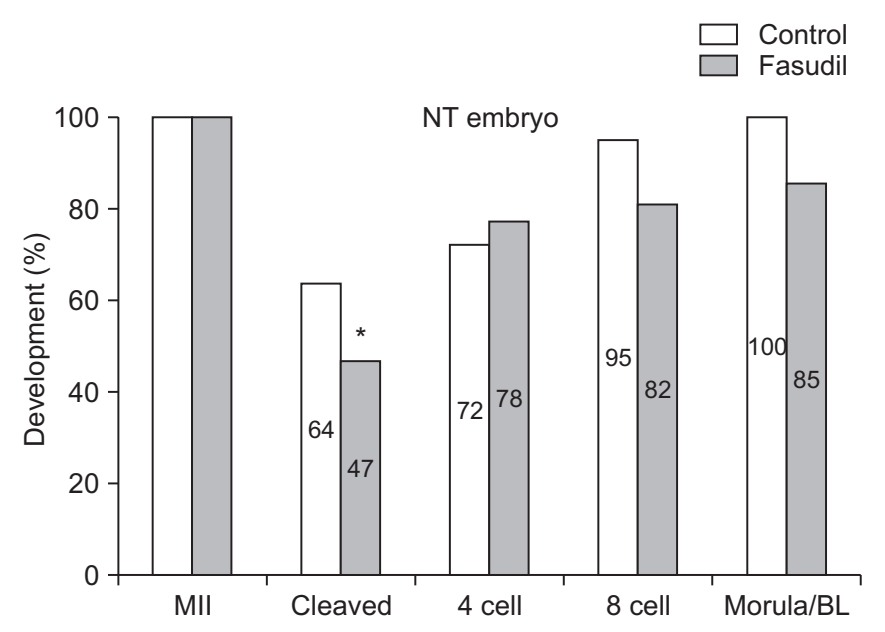

Fig. 3. Developmental rate of SCNT embryos. Fasudil treated embryos tended to show lower development rates than untreated controls at each stage except 4 cell stages. Especially, 2 cell cleaved rate was significantly lower (98/207 vs. 29/45). Development (\%) was calculated based on the number of embryos developed at the previous stage of each stage $\left({ }^{*} p<0.05\right.$; versus control). Statistical significance ( $p$-value) was calculated using Fisher's exact test. SCNT; somatic cell nuclear transfer.

using MEFs, and NT-oocytes which were parthenogenetically activated by activation medium supplemented with CCB. After activation, oocytes with PN were selected and cultured. A total of 45 oocytes in the fasudil free control group and 207 oocytes in the fasudil treated group were performed. In the control group, 20 of 45 NT-embryos developed to blastocysts (success rate 44\%), but only 53 of 207 NT-embryos (success rate 26\%) developed to blastocysts when treated with fasudil (Fig. 2). After fasudil was treated, the rate of 2 cell cleavage was significantly lower than the control while it did not affect the development up to morula/blastocyst (Fig. 3). These results suggest that fasudil is not suitable for use in embryo development.

\section{Fasudil increases the establishment of NT-mESCs}

Although fasudil negatively affects the embryo development into blastocysts, the rate of establishing mESCs from blastocysts could be improved. To investigate the effectiveness of fasudil, the zona pellucida of the blastocysts was removed using AT solution and seeded on the MEF

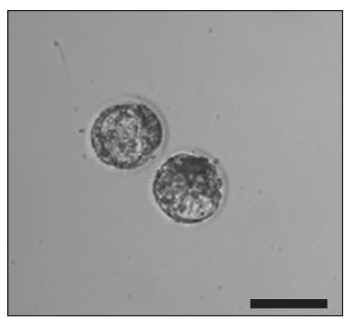

Bad-quality blastocysts

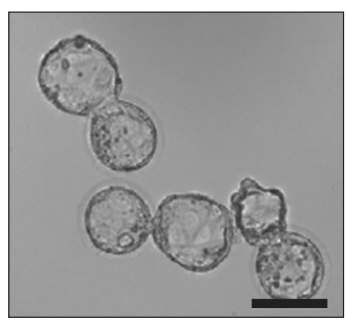

Good-quality blastocysts $\rightarrow$ No treatment Fasudil
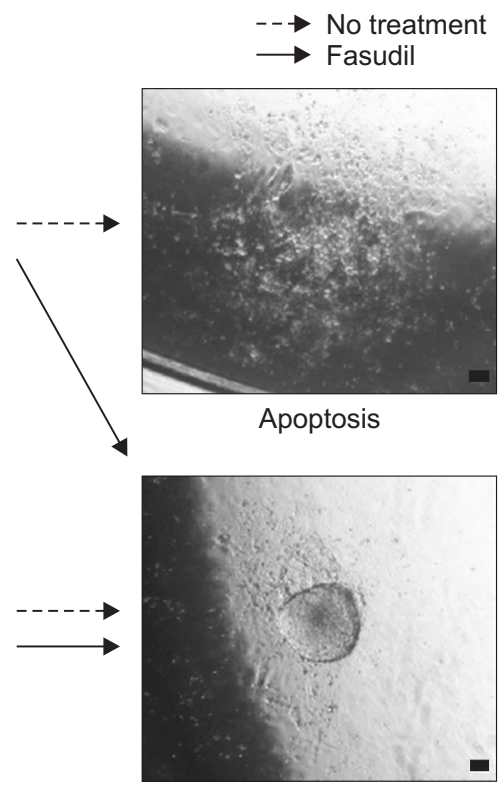

NT-mESCs
B
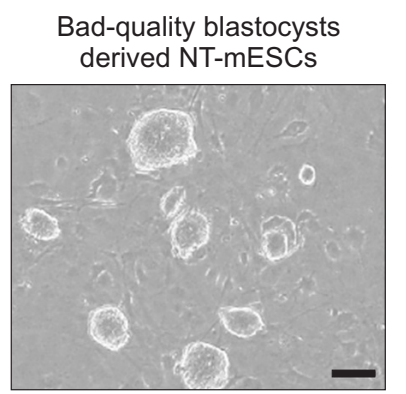

C

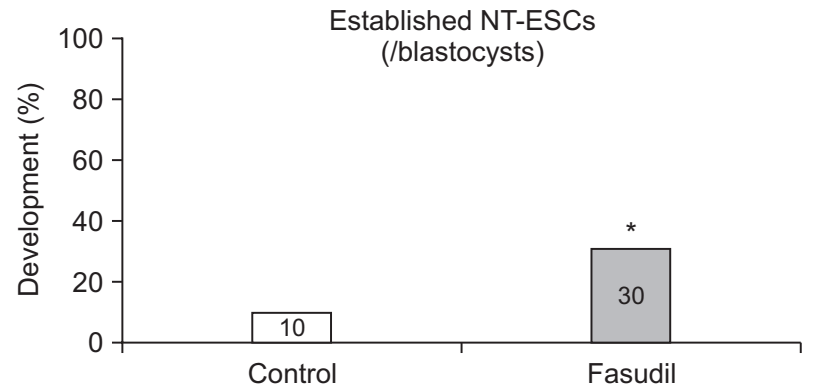

Fig. 4. Improvement of NT-ESC establishment rate by fasudil treatment. (A) Generally, bad-quality SCNT-blastocysts were not established ESCs. Bad quality blastocyst caused apoptosis when transferred to MEF without fasudil treatment. The blastocysts were divided into good and bad blastocysts according to the following criteria of the Gardner blastocyst grading system: Inner cell mass quality; A: many cells, tightly packed, B: several cells, loosely grouped, C: very few cells, Trophectoderm quality; A: many cells, forming a cohesive layer, B: few cells, forming a loose epithelium, C: very few large cells. (B) NT-mESC morphology derived from bad- and good-quality blastocysts. mESCs derived from bad-quality blastocysts were identical to the morphology of mESCs derived from good-quality blastocysts. Scale bar $=100 \mu \mathrm{m}$. (C) Efficiency of NT-mESCs establishment with or without fasudil. Fasudil increased the establishment rate of NT-mESCs (16/53 vs. $2 / 20)\left({ }^{*} p<0.05\right.$, versus control). Statistical significance ( $p$-value) of the results were calculated using Fisher's exact test. NT-mESCs; nuclear transfer mouse embryonic stem cells. 
feeder cells. In the process, the blastocysts were divided into two groups ased on their quality.

The good-quality blastocysts, which graded as AA and $A B$, were established mESCs regardless of fasudil treatment, but small and bad-quality blastocysts, graded as $\mathrm{BA}, \mathrm{BB}, \mathrm{BC}, \mathrm{CB}$, and $\mathrm{CC}$, were established mESCs rarely in the absence of fasudil. The bad-quality blastocysts have undergone apoptosis following transfer onto MEF coated culture plates without fasudil (Fig. 4A). On the other hand, the fasudil treated bad-quality blastocyst developed to NT-mESCs, which is identical in morphology to the dome shape of NT-mESCs derived from the good-quality blastocyst (Fig. 4B). When fasudil was treated to the culture medium, $30 \%(16 / 53)$ of the blastocysts developed to mESCs, but only $10 \%(2 / 20)$ of the untreated group's cells survived (Fig. 4C). In summary, fasudil significantly increased the establishment of NT-ESCs from the blastocysts in the mouse.

\section{DISCUSSION}

The ESCs are useful tools for a variety of studies such as regenerative medicine, embryology, and drug development. However, the embryos derived from nuclear transfer have low developmental potency to ESCs due to many factors such as '2-cell block' and apoptosis. Among them, '2-cell block' is reported to occur more in NT-embryo than in normal embryos (Gao et al., 2003; Wakayama, 2003). Also, ESCs derived from in vitro fertilized (IVF) embryos have limitations in therapeutic studies because they are genetically divergent from the patient (allogenic). SCNT-derived ESCs, on the other hand, can be used for various studies because they are identical to the genotype of the patient.

The first introduced ROCK inhibitor Y-27632 is widely used in the field of PSCs as a result of its ability to increase the proliferation of various cell types by preventing apoptosis. However, the synthesis of Y-27632 consists of seven stages, and the overall yield is only $45 \%$, which leads to a rise in product prices. (Paleček et al., 2011). In addition, chiral molecules such as Y-27632 do not recommend for the development of drugs due to the formation of an isomeric structure that can cause unpredictable side effects such as abnormality. On the other hand, since the Y-27632, there are various ROCK inhibitors were discovered, such as fasudil, ripasudil, and RKI-1447 (LIU Ai-jun et al., 2011). Among them, fasudil also increases the survival of many types of cells (Cavallini et al., 2003; Chen Meihui et al., 2013) and approved for clinical use in Japan and China (LIU Ai-jun et al., 2011; Liu Guang Jian et al., 2012).

Hence we were expecting that fasudil might have positive effects at the overall developmental stages up to the NT-mESC. However, contrary to the hypothesis, our findings showed that fasudil has different effects on embryos according to developmental stages. It negatively affected the preimplantation embryo development in vitro because the cleavage rate was reduced at the 2 cell stage. On the other hand, there was no difference between the groups at the 4 cell, 8 cell, and morula/BL stages. It is known that the PI3 kinase/AKT pathway must be activated for development during the zygote to 2 cell stage (Jin et al., 2009), and ROCK is known to activate the AKT pathway by inducing the activation of PI3 kinase (Jiao et al., 2017). When fasudil inhibits ROCK, it also causes inhibition on the AKT pathway. Through this mechanism, fasudil might induce to decline in the development of NT-zygotes.

Additionally, the effect of fasudil on NT-mESC establishment investigated at the stage from blastocyst to mESCs. In this stage, we divided the blastocysts into a group of the good-quality blastocysts with Gardner grades $\mathrm{AA}$ and $\mathrm{AB}$, and a group of the bad-quality blastocysts with $\mathrm{BA}, \mathrm{BB}, \mathrm{BC}, \mathrm{CB}$, and $\mathrm{CC}$ grades (Gardner et al., 2000). Under normal conditions, bad-quality blastocysts are not able to survive and develop into mESCs because of apoptosis, but after the treatment of fasudil, a significant number of bad-quality blastocysts survived and developed into mESCs. Furthermore, the rate of establishment from good-quality blastocysts to mESCs was also higher when supplemented with fasudil. During the development of mESC from blastocysts, apoptosis is induced by the ROCK signal, and fasudil increases the survival of mESCs by inhibiting ROCK signal (Coleman et al., 2001). In the process of NT-ESC establishment, the zona pellucida of blastocysts in AT solution should be removed, and ICM of blastocyst should be transferred to ESC media. This process might be noticeably stressful for blastocysts. However, our study showed that fasudil might play a role in protecting blastocyst from various stresses that cause apoptosis. 


\section{CONCLUSION}

To determine the possible utilization of fasudil in the developmental stage of the embryo after SCNT, we performed NT using hormonal ovulated MII oocytes and MEFs and added fasudil to the culture of embryos after parthenogenetic activation. The fasudil negatively affected the rate of development up to the blastocyst. However, in the stage from blastocysts to mESCs, it significantly increased the rate of $\mathrm{mESC}$ establishment. These results suggest that fasudil might not be suitable for the preimplantation embryo development, but it has effects on enhancing mESC establishment from blastocysts.

\section{CONFLICTS OF INTEREST}

No potential conflict of interest relevant to this article was reported.

\section{ACKNOWLEDGEMENTS}

We thank the Laboratory of Animal Research at Asan Medical Center for their technical support. The study was supported by grants from the National Research Foundation of Korea (NRF-2018R1A2B3001244).

\section{AUTHOR CONTRIBUTIONS}

S So and E Kang conceived the study and designed the experiments. E Kang performed the somatic cell nuclear transfer. Y Lee carried out the artificial activation of oocyte and embryo culture. S So and Y Lee performed cell cultivation. S So prepared materials related to the experiment and performed hormone injection. S So, Y Lee, and E Kang analyzed data. S So, MZ Karagozlu, and E Kang wrote the manuscript. All authors provided critical feedback and helped shape the research, analysis, and manuscript.

\section{AUTHOR'S POSITION AND ORCID NO.}

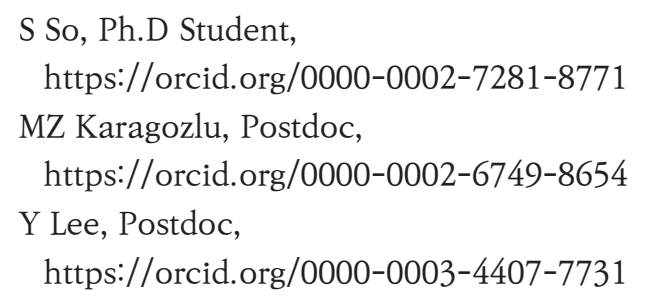

E Kang, Professor, https://orcid.org/0000-0002-2830-6779

\section{REFERENCES}

Amano M, Nakayama M, Kaibuchi K. 2010. Rho-kinase/ROCK: a key regulator of the cytoskeleton and cell polarity. Cytoskeleton. 67:545-554.

Cavallini A, Micieli G, Marcheselli S, Quaglini S. 2003. Role of monitoring in management of acute ischemic stroke patients. Stroke. 34:2599-2603.

Chen M, Liu A, Ouyang Y, Huang Y, Chao X, Pi R. 2013. Fasudil and its analogs: a new powerful weapon in the long war against central nervous system disorders? Expert Opinion on Investigational Drugs. 22:537-550.

Chen S, Luo M, Zhao Y, Zhang Y, He M, Cai W, Liu A. 2015. Fasudil stimulates neurite outgrowth and promotes differentiation in C17. 2 neural stem cells by modulating notch signalling but not autophagy. Cellular Physiology and Biochemistry. 36:531-541.

Cheng Y-T, Yeih D-F, Liang S-M, Chien C-Y, Yu Y-L, Ko B-S, Jan Y-J, Kuo C-C, Sung L-Y, Shyue S-K. 2015. Rho-associated kinase inhibitors promote the cardiac differentiation of embryonic and induced pluripotent stem cells. International Journal of Cardiology. 201:441-448.

Coleman ML, Sahai EA, Yeo M, Bosch M, Dewar A, Olson MF. 2001. Membrane blebbing during apoptosis results from caspase-mediated activation of ROCK I. Nature Cell Biology. 3:339.

Gao S, McGarry M, Latham KE, Wilmut I. 2003. Cloning of mice by nuclear transfer. Cloning \& Stem Cells. 5:287-294.

Gardner DK, Lane M, Stevens J, Schlenker T, Schoolcraft WB. 2000. Blastocyst score affects implantation and pregnancy outcome: towards a single blastocyst transfer. Fertility and Sterility. 73:1155-1158.

Hu Y, Li X, Huang G, Wang J, Lu W. 2019. Fasudil may induce the differentiation of bone marrow mesenchymal stem cells into neuron-like cells via the Wnt/ $\beta$-catenin pathway. Molecular Medicine Reports. 19:3095-3104.

Jiao X, Ashtari N, Rahimi-Balaei M, Min Chen Q, Badbezanchi I, Shojaei S, Marzban A, Mirzaei N, Chung S, Guan T. 2017. Mevalonate cascade and neurodevelopmental and neurodegenerative diseases: Future targets for therapeutic application. Current Molecular Pharmacology. 10:115-140.

Jin X, Chandrakanthan V, Morgan H, O'neill C. 2009. Preimplantation embryo development in the mouse requires the latency of TRP53 expression, which is induced by a ligandactivated PI3 kinase/AKT/MDM2-mediated signaling pathway. Biology of Reproduction. 80:286-294.

Kang E, Wu G, Ma H, Li Y, Tippner-Hedges R, Tachibana M, Sparman M, Wolf DP, Schöler HR, Mitalipov S. 2014. Nuclear reprogramming by interphase cytoplasm of two-cell mouse embryos. Nature. 509:101-104.

Kawase E, Yamazaki Y, Yagi T, Yanagimachi R, Pedersen RA. 
2000. Mouse embryonic stem (ES) cell lines established from neuronal cell-derived cloned blastocysts. Genesis. 28:156163.

Leyva V, Buckrell B, Walton J. 1998. Follicular activity and ovulation regulated by exogenous progestagen and PMSG in anestrous ewes. Theriogenology. 50:377-393.

Liu A-j, Ling F, Wang D, Wang Q, LÜ X-d, Liu Y-1. 2011. Fasudil inhibits platelet-derived growth factor-induced human pulmonary artery smooth muscle cell proliferation by upregulation of p27kipl via the ERK signal pathway. Chinese Medical Journal. 124:3098-3104.

Liu GJ, Wang ZJ, Wang YF, Xu LL, Wang XL, Liu Y, Luo GJ, He GH, Zeng YJ. 2012. Systematic assessment and meta-analysis of the efficacy and safety of fasudil in the treatment of cerebral vasospasm in patients with subarachnoid hemorrhage. European Journal of Clinical Pharmacology. 68:131-139.

Ma H, Morey R, O'Neil RC, He Y, Daughtry B, Schultz MD, Hariharan M, Nery JR, Castanon R, Sabatini K. 2014. Abnormalities in human pluripotent cells due to reprogramming mechanisms. Nature. 511:177.

Moon J, Jung M, Roh S. 2017. Comparison of developmental efficiency of murine somatic cell nuclear transfer protocol. Journal of Animal Reproduciton and Biotechnology. 32:8186.

Neev J, Gonzalez A, Licciardi F, Alikani M, Tadir Y, Berns M, Cohen J. 1993. Opening of the mouse zona pellucida by laser without a micromanipulator. Human Reproduction. 8:939944.

Paleček J, Zweigerdt R, Olmer R, Martin U, Kirschning A, Dräger G. 2011. A practical synthesis of Rho-Kinase inhibitor Y-27632 and fluoro derivatives and their evaluation in human pluripotent stem cells. Organic \& Biomolecular Chemistry. 9:5503-5510.
Polejaeva I, Campbell K. 2000. New advances in somatic cell nuclear transfer: application in transgenesis. Theriogenology. 53:117-126.

Roh S. 2012. Somatic cell nuclear transfer in rodents, the little big animals. Journal of Animal Reproduciton and Biotechnology. 27:205-209.

So S, Lee Y, Park J, Lee JY, Kim D, Hwang J, Shin J, Choi J, Han Y, Kang S, Dutton JR, Seo EJ, Lee BH, Kim CJ, Mitalipov S, Oh S, Kang E. 2019. The Rho-associated kinase inhibitor fasudil can replace Y-27632 for use in human pluripotent stem cell research. Preprints. doi: 10.20944.

Tachibana M, Amato P, Sparman M, Gutierrez NM, TippnerHedges R, Ma H, Kang E, Fulati A, Lee H-S, Sritanaudomchai H. 2013. Human embryonic stem cells derived by somatic cell nuclear transfer. Cell 153:1228-1238.

Tsung H, Mummery CL. 1990. Effects of feeder layer and BRL conditioned medium on mouse embryonic stem cells. Cell Research. 1:35.

Wakayama T. 2003. Cloned mice and embryonic stem cell lines generated from adult somatic cells by nuclear transfer. Oncology Research Featuring Preclinical and Clinical Cancer Therapeutics. 13:309-314.

Watanabe K, Ueno M, Kamiya D, Nishiyama A, Matsumura M, Wataya T, Takahashi JB, Nishikawa S, Nishikawa S-i, Muguruma K. 2007. A ROCK inhibitor permits survival of dissociated human embryonic stem cells. Nature Biotechnology. 25:681.

Ying QL, Wray J, Nichols J, Batlle-Morera L, Doble B, Woodgett J, Cohen P, Smith A. 2008. The ground state of embryonic stem cell self-renewal. Nature. 453:519-523.

Zhao C, Yao R, Hao J, Ding C, Fan Y, Dai X, Li W, Hai T, Liu Z, Yu Y. 2007. Establishment of customized mouse stem cell lines by sequential nuclear transfer. Cell Research. 17:80. 